\title{
Componentes de rendimento em genótipos de sorgo sacarino
}

\author{
Yield components in sweet sorghum genotypes \\ Componentes del rendimiento en genotipos de sorgo dulce
}

Recebido: 10/05/2021 | Revisado: 15/05/2021 | Aceito: 16/05/2021 | Publicado: 19/05/2021

Taniele Carvalho de Oliveira

ORCID: https://orcid.org/0000-0002-6900-6449 Universidade do Estado de Mato Grosso, Brasil

E-mail: taniele.carvalho@unemat.br

Altacis Junior de Oliveira

ORCID: https://orcid.org/0000-0002-6787-7160

Universidade do Estado de Mato Grosso, Brasil

E-mail: juniormarque11@gmail.com

Mirian da Silva Almici

ORCID: https://orcid.org/0000-0001-9467-7060

Universidade do Estado de Mato Grosso, Brasil

E-mail: mirianalmici@hotmail.com

Andressa Alves Cabreira dos Santos

ORCID: https://orcid.org/0000-0001-6731-9575

Universidade do Estado de Mato Grosso, Brasil

E-mail: acabreyra@gmail.com

Valvenarg Pereira da Silva

ORCID: https://orcid.org/0000-0001-8450-3016 Universidade do Estado de Mato Grosso, Brasil

E-mail: silvabiologo@hotmail.com

Anelise Saldanha Carneiro Pires

ORCID: https://orcid.org/0000-0002-4475-5742

Universidade do Estado de Mato Grosso, Brasil

E-mail: anelisesaldanha2016@gmail.com

Lucas Henrique Pereira Morais

ORCID: https://orcid.org/0000-0002-3251-5821

Universidade do Estado de Mato Grosso, Brasil

E-mail: lucas.morais@unemat.br

Júlio César Costa Rodrigues

ORCID: https://orcid.org/0000-0001-9387-7888 Universidade do Estado de Mato Grosso, Brasil

E-mail: Juliocesarc.r18@gmail.com

Marco Antonio Aparecido Barelli

ORCID: https://orcid.org/0000-0002-6385-6733

Universidade do Estado de Mato Grosso, Brasil

E-mail: mbarelli@unemat.br

Flávio Dessaune Tardin

ORCID: https://orcid.org/0000-0001-5755-2728

Empresa Brasileira de Pesquisa Agropecuária - Milho e Sorgo, Brasil

E-mail: flavio.tardin@embrapa.br

\begin{abstract}
Resumo
O sorgo sacarino assemelha-se à cana-de-açúcar por apresentar colmos suculentos e altos teores de açúcares fermentescíveis, a cultura é uma fonte renovável capaz de contribuir para o aumento da produção de etanol na entressafra canavieira. Desta forma, o sorgo sacarino torna-se uma cultura complementar na usina, a fim de manter e/ou aumentar a oferta de etanol no mercado. Diante do exposto, objetivou-se com o presente trabalho avaliar o potencial agronômico de genótipos de sorgo sacarino baseado em características morfoagronômicas. Foram avaliados 25 genótipos de sorgo sacarino em delineamento de blocos casualizados, com três repetições, avaliando nove características morfoagronômicas. Os dados foram submetidos à análise de variância e ao teste de Scott-Knott a 1\% de probabilidade, também foram estimados os coeficientes de correlação entre as características, pelo coeficiente de correlação de Pearson. Com base nos resultados, pode-se concluir que existe variabilidade entre os genótipos de sorgo sacarino para as características morfoagronômicas avaliadas. Os genótipos CMSXS642, CMSXS646 e CMSXS647 apresentaram melhor desempenho em relação às características avaliadas, mostrando-se bastante promissores para a produção de etanol.
\end{abstract}

Palavras-chave: Características morfoagronômicas; Produção de etanol; Sorghum bicolor L. Moench. 


\begin{abstract}
The sweet sorghum resembles sugar cane because it has succulent stems and high levels of fermentable sugars, the crop is a renewable source capable of contributing to the increase of ethanol production in the inter-harvest sugarcane. In this way, the sweet sorghum becomes a complementary crop in the plant, in order to maintain and/or increase the ethanol supply in the market. In view of the above, this study aimed to evaluate the agronomic potential of sweet sorghum genotypes based on morphoagronomic characteristics. Twenty-five genotypes of sweet sorghum were evaluated in a randomized block design, with three repetitions, evaluating nine morphoagronomic characteristics. The data were submitted to analysis of variance and the Scott-Knott test at $1 \%$ probability, the correlation coefficients between the characteristics were also estimated by Pearson's correlation coefficient. Based on the results, it can be concluded that there is variability among the genotypes of sweet sorghum for the morphoagronomic characteristics evaluated. The genotypes CMSXS642, CMSXS646 and CMSXS647 performed better in relation to the characteristics evaluated, showing great promise for ethanol production.
\end{abstract}

Keywords: Morphoagronomic characteristics; Ethanol production; Sorghum bicolor L. Moench.

\title{
Resumen
}

El sorgo dulce se parece a la caña de azúcar porque tiene tallos suculentos y altos niveles de azúcares fermentables, el cultivo es una fuente renovable capaz de contribuir al aumento de la producción de etanol entre las cosechas. De esta manera, el sorgo dulce se convierte en un cultivo complementario en la fábrica para mantener y/o aumentar la oferta de etanol en el mercado. En vista de lo anterior, este estudio tuvo como objetivo evaluar el potencial agronómico de los genotipos de sorgo dulce en base a las características morfo-agronómicas. Se evaluaron 25 genotipos de sorgo dulce en un diseño de bloques aleatorios, con tres repeticiones, evaluando nueve características morfoagronómicas. Los datos se sometieron al análisis de la varianza y a la prueba de Scott-Knott con una probabilidad del $1 \%$, los coeficientes de correlación entre las características también se estimaron mediante el coeficiente de correlación de Pearson. Sobre la base de los resultados, se puede concluir que existe variabilidad entre los genotipos de sorgo dulce para las características morfoagronómicas evaluadas. Los genotipos CMSXS642, CMSXS646 y CMSXS647 se comportaron mejor en relación con las características evaluadas, mostrando una gran promesa para la producción de etanol.

Palabras clave: Características morfo-agronómicas; Producción de etanol; Sorgo bicolor L. Moench.

\section{Introdução}

O sorgo sacarino (Sorghum bicolor L. Moench) é uma cultura energética promissora devido ao armazenamento de açúcares fermentescíveis (sacarose, glicose e frutose) nos colmos assemelhando-se à cana-de-açúcar, sendo estes facilmente convertidos em etanol, além de potencial para uso na produção de forragem (Parrella, 2011; Khalil et al., 2015). A cultura se adapta ao setor sucroalcooleiro, principalmente quando cultivado no verão, visando fornecer matéria-prima de qualidade para abastecer o mercado na entressafra da cana-de-açúcar, de forma a reduzir a instabilidade do mercado de etanol no Brasil (May et al., 2013).

O cultivo oferece ao produtor algumas vantagens como a rapidez no ciclo, cultura totalmente mecanizável, produção de grãos em torno de 2,5 t ha-1, produção de biocombustível, utilização do bagaço como fonte de energia e produção de forragem para alimentação de animais (Parrella, 2011). Além do fornecimento de matéria-prima para destilarias, pois apresenta grande quantidade de açúcares, o que faz dele uma fonte de açúcar e álcool, cerca de $8 \%$ inferior à da cana-de-açúcar, com o ${ }^{\circ}$ brix variando de 16 até 23\% e biomassa entre 40-70 t ha ${ }^{-1}$ (Olivetti \& Camargo, 1997; Almodares \& Hadi, 2009).

O Brasil é um dos maiores produtores de etanol do mundo e praticamente a totalidade deste combustível é gerada tendo-se a cana-de-açúcar como matéria prima. Na safra 2020/21 o país registrou um total de 32,9 bilhões de litros provenientes da cana-de-açúcar e do milho. Desse total, 10,5 bilhões de litros correspondem à produção do etanol anidro e 22,4 bilhões do etanol hidratado (CONAB, 2020).

Nesse contexto, o sorgo sacarino tem sido apontado como uma das matérias-primas renováveis capaz de contribuir para o aumento da competitividade do etanol brasileiro, seja para uso como cultura complementar à cana-de-açúcar e/ou para plantio em áreas consideradas marginais para a cana, seja para áreas que não tenham sido contempladas no zoneamento de riscos climáticos (Del Aguila et al., 2020). Dessa forma, a cultura atenderá a demanda de etanol no mercado devido ao seu 
potencial, além de satisfazer a demanda da usina, pois terão matéria-prima para a produção de etanol na entressafra, reduzindo o tempo ocioso e os custos de produção.

As usinas têm a possibilidade de iniciar suas atividades industriais com o sorgo sacarino a partir da segunda quinzena de março, visto que a janela ideal de plantio de sorgo é de 15 de novembro a 15 de janeiro, período em que essa cultura encontra as melhores condições de desenvolvimento, proporcionando, assim, um aumento na amplitude da janela de colheita. O potencial mínimo de geração de etanol de sorgo sacarino está estimado em $3.223 \mathrm{~L} \mathrm{ha}^{-1}$, com uma produtividade que gira em torno de $55 \mathrm{t} \mathrm{ha}^{-1}$, o que representa cerca de 58,6 L t (Assis \& Moraes, 2014).

Diversos experimentos têm mostrado as potencialidades do sorgo sacarino para produção de etanol (Giacomini et al., 2013; Nascimento et al., 2017; Batista et al., 2018; Câmara et al., 2018; Silva et al., 2020). Entretanto, o setor sucroenergético brasileiro tem sofrido graves crises em função da oscilação no preço do etanol devido à sazonalidade na produção e às variações climáticas, além do período de entressafra da cana-de-açúcar, que resulta em elevada ociosidade das unidades industriais.

Desta forma, o sorgo sacarino torna-se uma cultura complementar na usina, a fim de manter e/ou aumentar a oferta de etanol no mercado. Diante do exposto, objetivou-se com o presente trabalho avaliar o potencial agronômico de genótipos de sorgo sacarino baseado em características morfoagronômicas.

\section{Metodologia}

O ensaio foi conduzido na área experimental do Laboratório de Recursos Genéticos \& Biotecnologia (LRG\&B), na Universidade do Estado de Mato Grosso (UNEMAT), no município de Cáceres - MT (latitude de 1604’59” Sul e longitude 57³9’01”' Oeste), com altitude de $118 \mathrm{~m}$, em um Latossolo vermelho amarelo distrófico.

O clima da região é classificado como tropical megatérmico sub-úmido, com as temperaturas médias anuais entre $25^{\circ} \mathrm{C}$ e $26^{\circ} \mathrm{C}$, enquanto as máximas ultrapassam, frequentemente, $35^{\circ} \mathrm{C}$ durante quase todo o ano, e o período seco se prolonga de abril-maio a setembro-outubro, em geral. E com precipitação média anual de aproximadamente $104 \mathrm{~mm}$ (Sanches et al., 2020).

O plantio foi realizado na segunda quinzena do mês de dezembro de 2012, que coincide com o período chuvoso na região. Foram avaliados 25 genótipos de sorgo sacarino cedidos pelo Programa de Melhoramento Genético da Empresa Brasileira de Pesquisa Agropecuária - EMBRAPA Milho e Sorgo, sendo parte deles cultivares comerciais e o restante cultivares experimentais: (1)BR501, (2)BR505, (3)BRS506, (4)BRS509, (5)CMSXS630, (6)CMSXS634, (7)CMSXS642, (8)CMSXS643, (9)CMSXS644, (10)CMSXS646, (11)CMSXS647, (12)CMSXS648, (13)201027013, (14)201027014, (15)201027015, (16)201027016, (17)201027017, (18)201027018, (19)201027019, (20)201027020, (21)BRS601, (22)Sugargraze, (23)V82391, (24)V82392 e (25)V82393.

O delineamento experimental utilizado foi em blocos ao acaso (DBC), com três repetições. Cada unidade experimental foi constituída por quatro linhas de cinco metros de comprimento, espaçados em $0,70 \mathrm{~m}$ entre si, sendo consideradas como área útil apenas as duas linhas centrais. Em cada linha foram distribuídas três gramas de sementes, o desbaste foi realizado dez dias após a emergência, onde as parcelas úteis ficaram com 90 plantas em cada linha, simulando uma população de 140.000 plantas ha $^{-1}$.

$\mathrm{O}$ preparo da área foi realizado por meio de gradagem e a adubação realizada com base da análise de solo e de acordo com a recomendação da cultura, aplicando $150 \mathrm{~kg} \mathrm{ha}^{-1}$ do formulado mineral 20-05-20 N- $\mathrm{P}_{2} 0_{5}-\mathrm{K}_{2} \mathrm{O}$ e $375 \mathrm{~kg} \mathrm{ha}^{-1}$ de $\mathrm{P}_{2} 0_{5}$ para plantio e a adubação de cobertura foi realizado aos 45 dias utilizando $89 \mathrm{~kg} \mathrm{ha}^{-1}$ de $\mathrm{N}$ (Tabela 1). 
Tabela 1. Características químicas do Latossolo vermelho amarelo distrófico da área experimental, coletado na profundidade de $0-20 \mathrm{~cm}^{1 /}$.

\begin{tabular}{|c|c|c|c|c|c|c|c|c|c|c|c|}
\hline & \multirow{3}{*}{$\begin{array}{c}\mathrm{pH} \\
\mathrm{H}_{2} \mathrm{O}\end{array}$} & \multirow{3}{*}{$\begin{array}{c}\mathrm{pH} \\
\mathrm{CaCl}_{2}\end{array}$} & \multicolumn{6}{|c|}{ Análise Química } & \multicolumn{3}{|c|}{ Análise Física } \\
\hline \multirow{2}{*}{ Camada } & & & $\mathrm{P}$ & $\mathrm{K}$ & $\mathrm{Ca}$ & $\mathrm{Mg}$ & $\mathrm{H}+\mathrm{Al}$ & M.O & Areia & Silte & $\overline{\text { Argila }}$ \\
\hline & & & $\mathrm{mg} \mathrm{dm}^{-3}$ & & & \multicolumn{2}{|c|}{$\mathrm{Cmol}_{\mathrm{c}} \mathrm{dm}^{-3}$} & $\mathrm{~g} \mathrm{dm}^{-3}$ & & \multicolumn{2}{|c|}{$\mathrm{g} \mathrm{kg}^{-1}$} \\
\hline $0-20$ & 5,40 & 5,90 & 4,7 & 77,1 & 3,0 & 0,8 & $0,0 \quad 2,3$ & 13,2 & 72,5 & 12,6 & 14,9 \\
\hline
\end{tabular}

${ }^{1 / A n a ́ l i s e ~ r e a l i z a d a ~ n o ~ I n s t i t u t o ~ F e d e r a l ~ d e ~ E d u c a c ̧ a ̃ o ~ C i e ̂ n c i a ~ e ~ T e c n o l o g i a ~ d e ~ M a t o ~ G r o s s o ~-~ C a m p u s ~ d e ~ C a ́ c e r e s-M T, ~ L a b o r a t o ́ r i o ~ d e ~ A n a ́ l i s e ~}$ de Solo, 2012. Fonte: Autores.

Durante o ciclo de cultivo os tratos culturais foram realizados de acordo com a necessidade da cultura. A colheita dos genótipos foi realizada na primeira quinzena de abril de 2013, quando os grãos apresentaram no estágio duro/farináceo (aproximadamente 113 dias após o plantio). As análises de extração de caldo foram realizadas com o auxílio de uma moenda elétrica (motor $1 \mathrm{KW}$ ), onde os colmos foram passados duas vezes para a extração do caldo.

As seguintes características morfoagronômicas foram avaliadas:

a. FLOR (florescimento): número de dias decorridos do plantio até a data em que $50 \%$ das plantas da parcela estavam com pelo menos as flores do terço superior da panícula liberando pólen;

b. ALT (altura de planta): altura média de 10 plantas da área útil da parcela, medida da base da planta até o ápice da panícula na época da colheita $(\mathrm{m})$;

c. NCH (número de colmos por hectare): número médio de colmos colhido de 10 plantas na área útil da parcela na época da colheita (colmo ha-1);

d. NMF (número de folhas): número médio de folhas de 10 plantas na área útil da parcela na época da colheita;

e. DMC (diâmetro de colmo): diâmetro médio de colmos colhido de 10 plantas na área útil da parcela, medido a 10 $\mathrm{cm}$ da superfície do solo com auxílio de paquímetro digital na época da colheita $(\mathrm{mm})$;

f. PMV (produção de massa verde): peso médio de 5 plantas inteiras, cortadas a $10 \mathrm{~cm}$ da superfície do solo (sem panículas) na época da colheita $\left(\mathrm{kg} \mathrm{ha}^{-1}\right)$;

g. PMS (produção da massa seca): peso médio de 5 plantas inteiras, cortadas a $10 \mathrm{~cm}$ da superfície do solo (sem panículas) desidratadas em estufa de aeração forçada, na época da colheita $\left(\mathrm{kg} \mathrm{ha}^{-1}\right)$, a $65^{\circ} \mathrm{C}$ por 72 horas.

h. VMC (volume médio do caldo): volume médio do caldo extraído de 8 plantas inteiras (sem panículas), em L ha-1, prensadas no moinho elétrico na época da colheita;

i. ${ }^{\circ}$ BRIX (teor de sólidos solúveis totais): determinado no caldo a porcentagem de sólidos solúveis totais (\%) por meio de refratômetro digital de leitura automática, na época da colheita.

Os dados obtidos foram tabulados e submetidos à análise de variância univariada, considerando o efeito de genótipo como fixo, as médias foram agrupadas pelo teste de Scott-Knott ao nível de 1\% de probabilidade. Também foram estimados os coeficientes de correlação entre as características, pelo coeficiente de correlação de Pearson, com o auxílio do software estatístico GENES (Cruz, 2013).

A classificação dos coeficientes de correlação de Pearson (r) é feita de acordo com sua magnitude, os valores variam entre -1 e 1 . O valor de $r=0$ expressa correlação nula; $0,30<|r|<0,60$, considerada interação média; $0,60<|r|<0,90$, forte e quando $|r|=1$ a correlação é perfeita, sugerindo dependência completa, positiva ou negativa entre as características (Santos et al., 2016). 


\section{Resultados e Discussão}

Por meio da análise de variância observam-se diferenças significativas a $(\mathrm{P}<0,01)$ de probabilidade pelo teste $\mathrm{F}$ para as variáveis: FLOR, ALTP, NMC, PMS, NMF, DMC e ${ }^{\circ}$ BRIX, e significativa a $(\mathrm{P}<0,05)$ para as variáveis PMV e VMC, evidenciando variabilidade genética entre os genótipos avaliados (Tabela 2).

Tabela 2. Quadrados médios, médias e coeficientes de variação (CV) das análises de variância para nove características avaliadas em genótipos de sorgo sacarino.

\begin{tabular}{lcccccccccc}
\hline \multirow{2}{*}{ FV } & \multirow{2}{*}{ GL } & \multicolumn{9}{c}{ QUADRADOS MÉDIOS ${ }^{1 /}$} \\
\cline { 3 - 10 } & & FLOR & ALTP & NMC & NMF & DMC & PMV & PMS & VMC & ${ }^{\circ}$ BRIX \\
\hline BLO & 2 & 6,97 & 0,00 & 0,03 & 3,41 & 0,00 & 0,14 & 0,81 & 0,35 & 0,02 \\
GEN & 24 & $74,94^{* *}$ & $0,25^{* *}$ & $0,05^{* *}$ & $3,39^{* *}$ & $0,06^{* *}$ & $0,29 *$ & $0,19^{* *}$ & $0,20^{*}$ & $0,12^{* *}$ \\
RES & 48 & 5,75 & 0,01 & 0,00 & 0,49 & 0,02 & 0,14 & 0,08 & 0,10 & 0,03 \\
\hline MÉDIA & - & 80,67 & 2,77 & 12,21 & 13,23 & 18,59 & 28,36 & 17,50 & 26844,16 & 16,51 \\
CV $(\%)$ & - & 2,97 & 5,04 & 2,05 & 5,33 & 7,51 & 17,19 & 21,22 & 19,72 & 8,91 \\
\hline
\end{tabular}

${ }^{1 /}$ FLOR = número de dias para o florescimento (dias); ALTP = altura média final de planta $(\mathrm{m}) ; \mathrm{NMC}=$ número médio de colmos; $\mathrm{NMF}=$ número médio de folhas; $\mathrm{DMC}=$ diâmetro médio do caule $(\mathrm{mm}) ; \mathrm{PMV}=$ peso de massa verde $\left(\mathrm{kg} \mathrm{ha}^{-1}\right) ; \mathrm{PMS}=$ peso de massa seca $(\mathrm{kg}$ ha $\left.{ }^{1}\right) ; \mathrm{VMC}=$ volume médio do caldo $\left(\mathrm{L} \mathrm{ha}^{-1}\right) ;{ }^{\circ} \mathrm{BRIX}=$ porcentagem de ${ }^{\circ}$ Brix do caldo $(\%)$.

** e* significativos ao nível de 1 e $5 \%$ de probabilidade, respectivamente, pelo teste F. Fonte: Autores (2013).

Os coeficientes de variação - CV (\%) apresentaram valores baixos, indicando boa condução experimental e pouca influência do ambiente. O menor coeficiente de variação observado foi para a característica FLOR, com valor de $2,97 \%$ e o PMS demonstrou o maior coeficiente de variação com 21,22\%. Esses resultados estão dentro de limites encontrados por outros autores avaliando também genótipos de sorgo, como Almeida Júnior et al. (2020), Silva et al. (2020) e Romagnoli et al. (2020).

De acordo com a classificação proposta por Pimentel Gomes (2009), os coeficientes de variação de FLOR, ALT, $\mathrm{NCH}, \mathrm{NMF}$, DMC e ${ }^{\circ} \mathrm{BRIX}$ podem ser considerados como baixos, PMV e VMC como médio e PMS como alto. Ainda assim, estes resultados demonstrar que houve pouca influência das variações experimentais não-controláveis, considerando-se os valores do coeficiente de variação experimental e a significância entre os genótipos de sorgo sacarino avaliado.

As médias das características para os 25 genótipos de sorgo sacarino avaliados são apresentadas na tabela 3. Para a característica FLOR foram formados cinco grupos distintos, os genótipos floresceram em média de 80 dias, sendo que os mais precoces floresceram entre os 71 e 75 dias (BRS506, CMSXS646, 201027015, 201027016 e 201027017), demonstrando variabilidade entre os genótipos para esta característica. Neste caso, a precocidade dos genótipos de sorgo sacarino permite mais ciclos de cultivo durante o ano, maior flexibilidade no manejo dos sistemas de produção e um rápido retorno do capital investido. Além de possibilitar o cultivo na entressafra da cana-de-açúcar suprindo a ociosidade das usinas de etanol.

Pesquisa realizada por Cunha \& Lima (2010), avaliando 29 genótipos de sorgo forrageiro em Canguaretama - RN, em termos de dias para o florescimento obtiveram média de 73,48 dias, já Tabosa et al. (2012) avaliando genótipos de sorgo forrageiros e silageiro em diferentes ambientes do semiárido, observaram que os genótipos de porte em torno de dois metros geralmente apresentaram ciclo de 60 a 70 dias para atingirem $50 \%$ de florescimento. Em ambas pesquisas as médias do número de dias para o florescimento foram próximas das médias deste trabalho. 
Tabela 3. Agrupamento das médias dos genótipos de sorgo sacarino pelo método de Scott-Knott, estimado a partir de nove características morfoagronômicas.

\begin{tabular}{|c|c|c|c|c|c|c|c|c|c|}
\hline GENÓTIPO & FLOR & ALTP & NMC & DMC & NMF & PMV & PMS & VMC & ${ }^{\circ}$ BRIX \\
\hline BR501 & $90,00 \mathrm{a}$ & $2,48 \mathrm{c}$ & $13,26 \mathrm{a}$ & $17,15 \mathrm{a}$ & $14,90 \mathrm{a}$ & $25,67 \mathrm{a}$ & $15,62 \mathrm{a}$ & $20562,77 \mathrm{a}$ & $17,33 \mathrm{a}$ \\
\hline BR505 & $77,00 \mathrm{c}$ & $3,03 \mathrm{a}$ & $12,53 \mathrm{a}$ & $19,69 \mathrm{a}$ & $13,56 \mathrm{a}$ & $27,87 \mathrm{a}$ & $17,92 \mathrm{a}$ & $29491,34 \mathrm{a}$ & $17,33 \mathrm{a}$ \\
\hline BRS506 & $75,67 \mathrm{~d}$ & $2,86 \mathrm{~b}$ & $12,23 \mathrm{a}$ & $18,00 \mathrm{a}$ & $12,56 \mathrm{a}$ & $25,93 \mathrm{a}$ & $16,23 \mathrm{a}$ & $24404,76 \mathrm{a}$ & $16,76 \mathrm{a}$ \\
\hline BRS509 & $82,67 \mathrm{~b}$ & $2,84 \mathrm{~b}$ & $13,00 \mathrm{a}$ & $17,37 \mathrm{a}$ & $13,93 \mathrm{a}$ & 30,17 a & $19,00 \mathrm{a}$ & $28300,86 \mathrm{a}$ & $17,30 \mathrm{a}$ \\
\hline CMSXS630 & $80,67 \mathrm{~b}$ & $2,78 \mathrm{~b}$ & $12,17 \mathrm{a}$ & $20,02 \mathrm{a}$ & $13,00 \mathrm{a}$ & $30,21 \mathrm{a}$ & $17,66 \mathrm{a}$ & $26623,37 \mathrm{a}$ & $17,06 \mathrm{a}$ \\
\hline CMSXS634 & $79,67 \mathrm{c}$ & $3,02 \mathrm{a}$ & $13,10 \mathrm{a}$ & $22,51 \mathrm{a}$ & $13,66 \mathrm{a}$ & 33,11 a & $21,21 \mathrm{a}$ & $27867,96 \mathrm{a}$ & $16,50 \mathrm{a}$ \\
\hline CMSXS642 & $78,33 \mathrm{c}$ & $3,21 \mathrm{a}$ & $13,13 \mathrm{a}$ & $16,75 \mathrm{a}$ & $12,83 \mathrm{a}$ & $26,71 \mathrm{a}$ & $17,31 \mathrm{a}$ & $24945,89 \mathrm{a}$ & $20,26 \mathrm{a}$ \\
\hline CMSXS643 & $82,00 \mathrm{~b}$ & $2,74 \mathrm{~b}$ & $12,37 \mathrm{a}$ & $19,38 \mathrm{a}$ & $13,26 \mathrm{a}$ & $28,14 \mathrm{a}$ & $16,88 \mathrm{a}$ & $28409,09 \mathrm{a}$ & $17,76 \mathrm{a}$ \\
\hline CMSXS644 & 85,33 a & $3,27 \mathrm{a}$ & $13,43 \mathrm{a}$ & $18,89 \mathrm{a}$ & $13,33 \mathrm{a}$ & $28,44 \mathrm{a}$ & $17,92 \mathrm{a}$ & $27435,06 \mathrm{a}$ & $16,30 \mathrm{a}$ \\
\hline CMSXS646 & $74,00 \mathrm{~d}$ & $3,08 \mathrm{a}$ & $11,97 \mathrm{a}$ & $19,21 \mathrm{a}$ & $12,90 \mathrm{a}$ & $30,34 \mathrm{a}$ & $17,70 \mathrm{a}$ & $27651,51 \mathrm{a}$ & $19,70 \mathrm{a}$ \\
\hline CMSXS647 & $79,00 \mathrm{c}$ & $2,92 \mathrm{~b}$ & $11,53 \mathrm{a}$ & $19,03 \mathrm{a}$ & $12,96 \mathrm{a}$ & $37,40 \mathrm{a}$ & $23,46 \mathrm{a}$ & $35606,06 \mathrm{a}$ & $14,96 \mathrm{~b}$ \\
\hline CMSXS648 & $78,33 \mathrm{c}$ & $2,88 \mathrm{~b}$ & $12,27 \mathrm{a}$ & $19,36 \mathrm{a}$ & $13,26 \mathrm{a}$ & 26,62 a & $15,28 \mathrm{a}$ & $31331,17 \mathrm{a}$ & $15,46 \mathrm{~b}$ \\
\hline 201027013 & $87,00 \mathrm{a}$ & $2,99 \mathrm{a}$ & $12,87 \mathrm{a}$ & $18,50 \mathrm{a}$ & $14,33 \mathrm{a}$ & $32,68 \mathrm{a}$ & $27,23 \mathrm{a}$ & $31872,29 \mathrm{a}$ & $16,93 \mathrm{a}$ \\
\hline 201027014 & $81,33 \mathrm{~b}$ & $2,42 \mathrm{~d}$ & $11,77 \mathrm{a}$ & $20,53 \mathrm{a}$ & $12,36 \mathrm{a}$ & $32,64 \mathrm{a}$ & $18,31 \mathrm{a}$ & $23809,52 \mathrm{a}$ & $17,96 \mathrm{a}$ \\
\hline 201027015 & $71,67 \mathrm{~d}$ & $2,24 \mathrm{~d}$ & $9,93 \mathrm{a}$ & $17,10 \mathrm{a}$ & $11,23 \mathrm{a}$ & $23,29 \mathrm{a}$ & $13,07 \mathrm{a}$ & $26028,13 \mathrm{a}$ & $13,36 \mathrm{~b}$ \\
\hline 201027016 & $74,33 \mathrm{~d}$ & $2,26 \mathrm{~d}$ & $10,87 \mathrm{a}$ & $17,25 \mathrm{a}$ & $12,13 \mathrm{a}$ & $18,61 \mathrm{a}$ & $12,03 \mathrm{a}$ & $18344,15 \mathrm{a}$ & $15,83 \mathrm{~b}$ \\
\hline 201027017 & $72,00 \mathrm{~d}$ & $2,29 \mathrm{~d}$ & $10,73 \mathrm{a}$ & $16,80 \mathrm{a}$ & $11,20 \mathrm{a}$ & $21,51 \mathrm{a}$ & $12,46 \mathrm{a}$ & $19913,42 \mathrm{a}$ & $13,33 \mathrm{~b}$ \\
\hline 201027018 & $78,00 \mathrm{c}$ & $2,63 \mathrm{c}$ & $10,20 \mathrm{a}$ & $17,98 \mathrm{a}$ & $11,16 \mathrm{a}$ & $25,32 \mathrm{a}$ & $14,80 \mathrm{a}$ & $23971,86 \mathrm{a}$ & $17,43 \mathrm{a}$ \\
\hline 201027019 & $79,67 \mathrm{c}$ & $2,37 \mathrm{~d}$ & $11,90 \mathrm{a}$ & $18,42 \mathrm{a}$ & $13,50 \mathrm{a}$ & $24,41 \mathrm{a}$ & $15,28 \mathrm{a}$ & $22673,16 \mathrm{a}$ & $13,90 \mathrm{~b}$ \\
\hline 201027020 & $81,33 \mathrm{~b}$ & $2,79 \mathrm{~b}$ & $11,77 \mathrm{a}$ & $18,25 \mathrm{a}$ & $13,16 \mathrm{a}$ & $29,22 \mathrm{a}$ & $17,57 \mathrm{a}$ & $33062,77 \mathrm{a}$ & $16,23 \mathrm{a}$ \\
\hline BRS601 & 85,33 a & $2,74 \mathrm{~b}$ & $12,50 \mathrm{a}$ & $18,95 \mathrm{a}$ & $13,30 \mathrm{a}$ & $30,34 \mathrm{a}$ & $16,92 \mathrm{a}$ & 33766,23 a & $14,80 \mathrm{~b}$ \\
\hline Sugargraze & 84,67 a & $2,85 \mathrm{~b}$ & $13,00 \mathrm{a}$ & $18,72 \mathrm{a}$ & $15,06 \mathrm{a}$ & $30,78 \mathrm{a}$ & $19,22 \mathrm{a}$ & $25432,90 \mathrm{a}$ & $16,56 \mathrm{a}$ \\
\hline V82391 & 85,00 a & $2,90 \mathrm{~b}$ & $13,20 \mathrm{a}$ & $17,48 \mathrm{a}$ & $14,60 \mathrm{a}$ & $27,18 \mathrm{a}$ & 16,36 a & $26461,04 \mathrm{a}$ & $15,40 \mathrm{~b}$ \\
\hline V82392 & 89,00 a & $2,63 \mathrm{c}$ & $13,00 \mathrm{a}$ & $18,29 \mathrm{a}$ & $14,33 \mathrm{a}$ & 28,83 a & $17,88 \mathrm{a}$ & $25703,46 \mathrm{a}$ & $17,20 \mathrm{a}$ \\
\hline V82393 & 84,67 a & $3,00 \mathrm{a}$ & $12,60 \mathrm{a}$ & $19,00 \mathrm{a}$ & $14,03 \mathrm{a}$ & $33,42 \mathrm{a}$ & $20,17 \mathrm{a}$ & $27435,06 \mathrm{a}$ & $17,13 \mathrm{a}$ \\
\hline
\end{tabular}

FLOR = número de dias para o florescimento (dias); $\mathrm{ALTP}=$ altura média final de planta $(\mathrm{m}) ; \mathrm{NMC}=$ número médio de colmos; $\mathrm{NMF}=$ número médio de folhas; $\mathrm{DMC}=$ diâmetro médio do caule $(\mathrm{mm}) ; \mathrm{PMV}=$ peso de massa verde $\left(\mathrm{kg} \mathrm{ha}^{-1}\right) ; \mathrm{PMS}=$ peso de massa seca $(\mathrm{kg}$ ha $\left.{ }^{1}\right) ; \mathrm{VMC}=$ volume médio do caldo $\left(\mathrm{L} \mathrm{ha}^{-1}\right) ;{ }^{\circ} \mathrm{BRIX}=$ porcentagem de ${ }^{\circ}$ Brix do caldo (\%). Fonte: Autores (2013).

A média dos genótipos para ALTP foi de 2,77 m, apresentando amplitude de 2,24 m para o genótipo 201027015 a 3,27 m para o genótipo CMSXS644, ocorrendo à formação de quatro grupos distintos. Os genótipos avaliados apresentaram aptidão quanto à colheita mecanizada por não apresentarem acamamento durante o ciclo de cultivo, sendo está uma característica de grande relevância na escolha da cultivar a ser plantada. Além disso, a escolha de plantas de sorgo sacarino de maior porte pode apresentar dupla aptidão tanto para a produção de etanol quanto de forragem.

Os valores médios obtidos para ALT foram próximos aos resultados encontrados por outros autores, Nascimento et al. (2017) avaliando o desempenho de cultivares de sorgo sacarino na região de Humaitá, AM, obtiveram altura média dos genótipos de 2,75 metros. Romagnoli et al. (2020) avaliando o desempenho agronômico de cultivares de sorgo sacarino em diferentes épocas de semeadura, no Noroeste do Estado de São Paulo, observaram que a altura média das plantas de 2,67 
metros.

Para as características NMC, DMC, NMF, PMV, PMS e VMC as médias dos genótipos formaram apenas um único grupo cada. Para a característica NMC o genótipo 201027015 apresentou menor número de colmos $(9,93)$ e o genótipo CMSXS644 maior número (13,43). Os valores observados para DMC variaram de 16,75 mm a 21,51 mm, sendo a média dos genótipos de 18,58 mm. Esta característica é diretamente influenciada pela densidade de plantas, espaçamentos menores e maior quantidade de entrenó por metro tendem a originar colmos de menores diâmetros.

A redução dessa variável pode tornar as plantas mais sensíveis ao acamamento e ao quebramento, afetando negativamente as operações de colheita da cultura e consequentemente reduzindo a produtividade e qualidade do produto final (Silva et al., 2014). De acordo com Oliveira et al. (2018) a seleção de genótipos com maiores diâmetros de colmo é indicada, uma vez que esta é diretamente relacionada à produção de caldo, característica de extrema importância no sorgo destinado a produção de etanol.

O NMF entre os genótipos foi de 13,22, sendo que o genótipo Sugargraze apresentou maior número médio $(15,06)$ e o genótipo 201027018 menor NMF $(11,16)$. Observa-se que para as características PMV e PMS médias de 28,36 e 17,50 kg ha-1 respectivamente. O PMV apresentou amplitude de $18,61 \mathrm{~kg} \mathrm{ha}^{-1}$ (201027016) a 37,40 $\mathrm{kg} \mathrm{ha}^{-1}$ (CMSXS647), para o PMS o genótipo 201027013 obteve maior peso médio $\left(27,23 \mathrm{~kg} \mathrm{ha}^{-1}\right)$ e o genótipo 201027016 o menor peso médio $\left(12,03 \mathrm{~kg} \mathrm{ha}^{-1}\right)$.

De acordo com Pereira Filho et al. (2013) o peso de massa verde reflete positivamente na produção de caldo, entretanto, o conteúdo de açúcares no caldo e, por consequência, a conversão em etanol é dependente da cultivar, dos fatores ambientais, época de colheita, eficiência de processos de transporte e de conversão industrial.

A média dos genótipos para o VMC foi de 26.844,16 $\mathrm{L} \mathrm{ha}^{-1}$, as maiores médias foram observadas pelo genótipo CMSXS647, com 35.606,06 L ha-1 , seguido do genótipo BRS 601 que produziu 33.766,23 L ha-1, já os genótipos 201027016, 201027017 e BR501 foram os que apresentaram menores médias, 18.344,15 L ha-1, 19.913,42 L ha-1 e 20.562,77 L ha', respectivamente. Os resultados deste trabalho foram superiores ao encontrados por Giacomini et al. (2013) avaliando o comportamento de 25 cultivares de sorgo sacarino na Região Central do Tocantins, encontraram média geral para o volume de caldo próxima a $11.000 \mathrm{~L} \mathrm{ha}^{-1}$.

Vale ressaltar que as médias da variável VMC observado neste trabalho está subestimado, uma vez que a maneira e o equipamento utilizados para extração do caldo possuem eficiência inferior àqueles utilizados nas usinas de açúcar e etanol. Entretanto, pelo fato de a metodologia aplicada ter sido a mesma para todos os genótipos, os valores obtidos servem para identificação de genótipos que se destacaram visando à produção de etanol. Segundo Cavalcante et al. (2017) a produção de caldo em genótipos de sorgo sacarino está diretamente relacionada a diâmetro de colmo, altura de planta e massa verde.

A característica ${ }^{\circ}$ BRIX obteve a formação de dois grupos, a porcentagem média entre os genótipos foi de 16,51\%, onde os genótipos 201027015 e 201027017 apresentaram menores valores de 13,3\% e o genótipo CMSXS642 apresentou-se superior com 20,26\%. Resultados superiores ao deste trabalho forma encontrados por Giacomini et al. (2013) avaliando o comportamento de 25 cultivares de sorgo sacarino na Região Central do Tocantins, obtiveram resultados superiores ao encontrado neste trabalho, onde a média dos genótipos foi de $21,12 \%$.

Entretanto, Nascimento et al. (2017) avaliando cultivares de sorgo sacarino na região de Humaitá, AM, obtiveram média dos genótipos para ${ }^{\circ}$ BRIX de $11,47 \%$. Segundo Kawahigashi et al. (2013), a característica ${ }^{\circ}$ Brix está correlacionado com o conteúdo total de açúcares, e desta forma, pode ser utilizado como parâmetro na seleção e desenvolvimento de genótipos de sorgo sacarino ricos em açúcar.

$\mathrm{Na}$ Tabela 4, observam-se as correlações de Pearson entre as características avaliadas, os resultados foram significativos e positivos para 21 das associações ao nível de $1 \%$ e $5 \%$ de probabilidade, desse total ocorreram 13 correlações médias e oito correlações fortes. As características FLOR x NMC e FLOR x NMF estão fortemente correlacionas entre sim, e 
FLOR x PMV e FLOR x PMS possuem correlações médias entre sim, a seleção de plantas a partir do maior número de dias para o florescimento resultará em ganhos para as características correlacionadas. Tôrres et al. (2014) caracterizando cultivares de sorgo sacarino no norte de Minas Gerais visando a produção de etanol, verificaram correlação positiva e baixa entre FLOR e PMV, sugerindo que, em média, plantas de maior ciclo apresentam maior PMV, esses resultados corroboram aos encontrados nesse trabalho.

Tabela 4 - Coeficientes de correlação fenotípica de Pearson $(r f)$ entre caracteres de genótipos de sorgo sacarino, estimado a partir de nove características morfoagronômicas.

\begin{tabular}{lllllllll}
\hline Caráter & ALTP & NMC & PMV & PMS & NMF & DMC & VMC \\
\hline FLOR & 0,26 & $0,72^{* *}$ & $0,44^{*}$ & $0,47^{*}$ & $0,80^{* *}$ & 0,11 & 0,21 & 0,22 \\
ALTP & & $0,65^{* *}$ & $0,58^{* *}$ & $0,60^{* *}$ & $0,42^{*}$ & 0,33 & $0,55^{* *}$ & $0,54^{* *}$ \\
NMC & & & $0,44^{*}$ & $0,49^{*}$ & $0,86^{* *}$ & 0,24 & 0,23 & $0,43^{*}$ \\
PMV & & & $0,87^{* *}$ & $0,44^{*}$ & $0,61^{* *}$ & $0,70^{* *}$ & 0,30 \\
PMS & & & & $0,51^{* *}$ & $0,45^{*}$ & $0,62^{* *}$ & 0,30 \\
NMF & & & & & 0,17 & 0,24 & 0,24 \\
DMC & & & & & & & 0,39 \\
VMC & & & & & & & & 0,16 \\
\hline
\end{tabular}

** e $*$ significativos a 1 e $5 \%$ de probabilidade pelo teste $\mathrm{t}$ respectivamente, os demais foram não significativos.

FLOR = número de dias para o florescimento (dias); ALTP = altura média final de planta $(\mathrm{m}) ; \mathrm{NMC}=$ número médio de colmos; $\mathrm{NMF}=$ número médio de folhas; $\mathrm{DMC}=$ diâmetro médio do caule $(\mathrm{mm}) ; \mathrm{PMV}=$ peso de massa verde $\left(\mathrm{kg} \mathrm{ha}^{-1}\right) ; \mathrm{PMS}=$ peso de massa seca $(\mathrm{kg}$ ha $\left.{ }^{1}\right) ; \mathrm{VMC}=$ volume médio do caldo $\left(\mathrm{L} \mathrm{ha}^{-1}\right) ;{ }^{\circ} \mathrm{BRIX}=$ porcentagem de ${ }^{\circ}$ Brix do caldo $(\%)$. Fonte: Autores (2013).

As correlações entre as características ALTP x NMC apresentou correlação forte, e ALTP x PMV, PMS, NMF, VMC e ${ }^{\circ}$ BRIX apresentaram correlações médias, ganhos poderão ser obtidos para essas características a partir da seleção de plantas mais altas. Já para as combinações entre NMC x NMF a correlação foi forte e para as combinações entre NMC x PMS, PMV e ${ }^{\circ}$ BRIX as correlações foram medianas, isso demonstra que, quanto maior o número de colmo por planta maior será o ganho para as características correlacionadas.

As características PMV x PMS, DMC e VMC estão fortemente correlacionadas entre si, e PMV x NMF a correlação foi média, a seleção de plantas com maior peso de massa verde, resultará em plantas com maior o peso de massa seca, diâmetro do colmo, volume de caldo e número de folha. E as correlações entre PMS x NMF, DMC e VMC apresentaram correlações médias, ganhos poderão ser obtidos para número de folha, diâmetro do colmo e volume de caldo a partir da seleção de plantas com maior peso de massa seca.

De acordo com Ribeiro et al. (2013), o ideótipo de sorgo sacarino deve apresentar alta produtividade de massa verde com alto percentual de sólidos solúveis totais, neste caso o teor de brix torna-se bastante útil na seleção de cultivares de sorgo sacarino. Os referidos autores observaram alta correlação e positiva com PMV e SST entre 25 cultivares de sorgo sacarino, estes resultados diferem dos encontrados neste trabalho. De acordo com Albuquerque et al. (2008), com a análise de correlação linear, pode-se decidir pela redução do número de características avaliadas em experimentos futuros, uma vez observada grande dependência entre as variáveis.

Considerando o conjunto de características agronômicas avaliadas, destacaram-se os genótipos CMSXS642, CMSXS646 e CMSXS647 pelo maior potencial agronômico para a produção de etanol, apresentando maiores médias para volume de caldo e porcentagem de ${ }^{\circ}$ brix. Estes resultados reforçam a ideia de que o cultivo de sorgo sacarino é uma importante 
alternativa para complementar a cana-de-açúcar, proporcionando a otimização das usinas e aumentando a eficiência das mesmas no período da entressafra, reduzindo custos de produção de etanol.

\section{Conclusão}

Existe variabilidade entre os genótipos de sorgo sacarino para as características morfoagronômicas analisadas. Os genótipos CMSXS642, CMSXS646 e CMSXS647 apresentaram melhor desempenho em relação à essas características, mostrando-se bastante promissores para a produção de etanol. Contudo, sugere-se para trabalhos futuros avaliação da Interação Genótipo-Ambiente dos genótipos.

\section{Agradecimentos}

À Fundação de Apoio à Pesquisa do Estado de Mato Grosso (FAPEMAT) e Coordenação de Aperfeiçoamento de Pessoal de Nível Superior (CAPES) - Código Financeiro 001 pela concessão de bolsa, e à Embrapa Milho e Sorgo pelo apoio na condução dos experimentos.

\section{Referências}

Albuquerque, C. J. B., Von Pinho, R. G., \& da Silva, R. (2008). Produtividade de híbridos de milho verde experimentais e comerciais. Bioscience Journal, $24(2)$.

Almeida Júnior, J. J., Smiljanic, K. B. A., Matos, F. S. A., Perozini, A. C., de Sousa, J. V. A., Junior, L. F. R., ... \& Liberato, P. V. (2020). Utilização de fertilizante agroecologicamente correto no sorgo granífero BRG 21320 em diferentes doses, plantado em segunda safra no Sudoeste Goiano. Brazilian Journal of Development, 6(11), 87904-87915.

Almodares, A., \& Hadi, M. R. (2009). Produção de bioetanol a partir do sorgo doce: uma revisão. Jornal Africano de Pesquisa Agrícola, 4 (9), $772-780$.

Assis, R. T., Morais, C. G. (2014). Sorgo sacarino, a segunda safra do etanol no Brasil. Instituto de Ciências da Saúde, Agrárias e Humanas (ISAH), Circular técnica 11 .

Batista, V. A. P., Batista, V. Z. P., Pimentel, L. D., de Barros, A. F., Moreira, T. D. S., \& Dias, L. A. D. S. (2018). Produção de açúcares no caldo de sorgo sacarino avaliado em duas épocas de corte. Revista Brasileira de Milho e Sorgo, 17(2), 263-273.

Camara, T. M. M., Parrella, R. D. C., \& Silva, H. D. E. (2018). Qualidade industrial e rendimento de etanol de genótipos de sorgo sacarino em planta e rebrota. Embrapa Milho e Sorgo-Artigo em periódico indexado (ALICE).

Cavalcante, T. J., Giongo, P. R., de Paula Barbosa, K., do Nascimento Júnior, L. F., Rodrigues, A. A., \& Vital, R. G. (2017). Características agronômicas de cultivares de sorgo sacarino em diferentes épocas na região do Sudoeste de Goiás, Brasil. Revista Espacios, 38(46), 26.

Conab - Companhia Nacional de Abastecimento. (2020). Acompanhamento da safra brasileira de cana-de-açúcar, v. 7 - Safra 2019/20. Brasília: Conab.

Cruz, C. D. (2013). Genes: a software package for analysis in experimental statistics and quantitative genetics. Acta Scientiarum. Agronomy, 35(3), 271-276.

Cunha, E. E., \& Lima, J. M. P. D. (2010). Caracterização de genótipos e estimativa de parâmetros genéticos de características produtivas de sorgo forrageiro. Revista Brasileira de Zootecnia, 39(4), 701-706.

Del Aguila, L S. H., Gehling, R. K., Caputo, T., \& Saavedra Del Aguila, J. (2020). Melhor arranjo de plantas para os genótipos de sorgo sacarino BR 506 e BR 511 visando à produção de etanol. Brazilian Journal of Animal and Environmental Research, 3(2), 648-653.

Santos, C. V., de Menezes, C. B., SILVA, C., da Silva, R. A., Mingote, M., \& Rocha, D. (2016). Correlações genotípicas entre características agronômicas de sorgo granífero (Sorghum bicolor (L.) Moench). In Embrapa Milho e Sorgo-Artigo em anais de congresso (ALICE). In: CONGRESSO NACIONAL DE MILHO E SORGO, 31., 2016, Bento Gonçalves. Milho e sorgo: inovações, mercados e segurança alimentar: anais. Sete Lagoas: Associação Brasileira de Milho e Sorgo, 2016.

Giacomini, I., Pedroza, M. M., Siqueira, F. L. T., Mello, S. Q. S., Cerqueira, F. B., \& Salla, L. (2013). Uso potencial de sorgo sacarino para a produção de etanol no estado do Tocantins. Revista Agrogeoambiental, 5(3).

Kawahigashi, H., Kasuga, S., Okuizumi, H., Hiradate, S., \& Yonemaru, J. I. (2013). Avaliação do teor de Brix e açúcar no suco do caule de variedades de sorgo. Grassland Science, 59 (1), 11-19.

Khalil, S. R. A. Abdelhafez, A. A. \& Amer, E. A. M. (2015). Evaluation of bioethanol production from juice and bagasse of some sweet sorghum varieties. Annals of Agricultural Sciences, 60(2), 317-324.

May, A., Mendes, S., da Silva, D. D., Parrella, R. D. C., de Miranda, R. A., da Silva, A. F., ... \& Schaffert, R. (2013). Cultivo de sorgo sacarino em áreas de reforma de canaviais. Embrapa Milho e Sorgo-Circular Técnica (INFOTECA-E). 
Research, Society and Development, v. 10, n. 6, e35310615965, 2021

(CC BY 4.0) | ISSN 2525-3409 | DOI: http://dx.doi.org/10.33448/rsd-v10i6.15965

Nascimento, M. F., Campos, M. C. C., da Silva, D. M. P., Mantovanelli, B. C., Gomes, R. P., da Costa Weckner, F., \& Jordão, H. W. C. (2017). Avaliação de cultivares de sorgo sacarino (Sorghum bicolor (L.) Moench) na região amazônica, Brasil. Nativa, 5(6), 381-385.

Oliveira, A. R., de Melo, R. F., Simões, W. L., \& dos Santos, J. M. R. (2018). Viabilidade econômica do cultivo consorciado de sorgo sacarino e feijão-caupi em barragem subterrânea. In Embrapa Semiárido-Artigo em anais de congresso (ALICE). In: Congresso Internacional das Ciências Agrárias, 3., 2018, João Pessoa. Ciência, tecnologia e desenvolvimento rural: compartilhando conhecimentos inovadores e experiências. João Pessoa: Instituto Internacional Despertando Vocações, 2018.

Olivetti, M. P. A., \& de Camargo, A. M. M. P. (1997). Aspectos econômicos e desenvolvimento da cultura do sorgo. Informações Econômicas-Governo do Estado de São Paulo Instituto de Economia Agrícola, 27, 35-48.

Parrella, R. A. C. (2011). Sorgo sacarino: melhoramento genético do sorgo sacarino. Revista Agroenergia, 3, 8-9.

Pereira Filho, I. A., Parrella, R. A. D. C., Moereira, J. A. A., May, A., Vander Fillipe, D. S., \& Cruz, J. C. (2013). Avaliação de cultivares de sorgo sacarino [Sorghum bicolor (L.) Moench] em diferentes densidades de semeadura visando a características importantes na produção de etanol. Revista Brasileira de Milho e Sorgo, 12(2), 118-127.

Pimentel Gomes, F. (2009). Curso de estatística experimental. Piracicaba: FEALQ, 451.

Ribeiro, P. D. O., Parrella, R. D. C., Schaffert, R. E., de Souza, V. F., da Silva, M. J., França, A., ... \& de Oliveira, M. S. (2014). Caracterização de cultivares de sorgo sacarino na região central de Minas Gerais visando a produção de etanol. In Embrapa Milho e Sorgo-Artigo em anais de congresso (ALICE). In: CONGRESSO INTERNACIONAL DE BIOENERGIA, 9., 2014. São Paulo. Anais... Curitiba: Porths Eventos, 2014.

Romagnoli, M. J., de Freitas, R. S., Albuquerque, C. J. B., Borges, W. L. B., Pereira, A. J., \& Zoz, A. (2020). Época de semeadura sobre cultivares de sorgo sacarino. Brazilian Journal of Development, 6(9), 72484-72497.

Sanches, J. C. M., Domingos, R. M. A., Carolina, A., Ribeiro, V. B., \& Carignani, G. (2020). mMpa de análise climática de cidades de pequeno e médio portes no contexto do Pantanal Mato-Grossense. Revista de Geografia (Recife), 37(1).

Silva, C., Silva, A. F. D., Vale, W. G. D., Galon, L., Petter, F. A., May, A., \& Karam, D. (2014). Interferência de plantas daninhas na cultura do sorgo sacarino. Bragantia, 73(4), 438-445.

Silva, P. C., da Silva, M. V., Pereira, A. D., da Costa, A. R., Giongo, P. R., de Abreu, J. P., \& Moraes, V. H. (2020). Produtividade e características biométricas de Sorgo Sacarino sob torta de Filtro. Brazilian Journal of Development, 6(10), 78212-78226.

Tabosa, J., Silva, F., Nascimento, M., Barros, A., Brito, A., \& Simplício, J. (2012). Genótipos de Sorgo Forrageiro no Semiárido de Pernambuco e AlagoasEstimativas de Parâmetros Genéticos de variáveis de Produção. In Embrapa Milho e Sorgo-Artigo em anais de congresso (ALICE). In: CONGRESSO NACIONAL DE MILHO E SORGO, 29., 2012, Águas de Lindóia. Diversidade e inovações na era dos transgênicos: resumos expandidos. Campinas: Instituto Agronômico; Sete Lagoas: Associação Brasileira de Milho e Sorgo, 2012.

Tôrres, T. B. C. F., PARRElla, R. D. C., Parrella, N. N. L. D., Portugal, A. F., de Souza, V. F., Schaffert, R. E., ... \& dos Santos, C. V. (2012). Caracterização de cultivares de sorgo sacarino no Norte de Minas Gerais visando a produção de etanol. In Embrapa Milho e Sorgo-Artigo em anais de congresso (ALICE). In: CONGRESSO NACIONAL DE MILHO E SORGO, 29., 2012, Águas de Lindóia. Diversidade e inovações na era dos transgênicos: resumos expandidos. Campinas: Instituto Agronômico; Sete Lagoas: Associação Brasileira de Milho e Sorgo, 2012. 